\title{
Psicooncología
}

ISSN: 1696-7240

\section{Diseño y análisis de propiedades psicométricas del Inventario de Estilos de Pensamiento en Respuesta al Cáncer (IEPRaC)}

\author{
Mariana Alejandra Sierra-Murguía ${ }^{1 *}$; Lucia González-Alcocer²; Gabriela Navarro-Contreras ${ }^{3}$; \\ Martha Leticia Guevara-Sanginés ${ }^{4}$; Ferrán Padrós-Blázquez ${ }^{5}$
}

Recibido: 26 de noviembre de 2020 / Aceptado: 1 de marzo de 2021

Resumen. Una de las variables que ha sido definida como mediadora para la respuesta emocional ante el cáncer es el estilo de pensamiento. En contextos patológicos (por ejemplo, rumiación en depresión), y también en contextos normativos, se ha sugerido que el estilo de pensamiento puede predecir el ajuste ante un estresor. Existen instrumentos para evaluar de manera independiente los estilos de pensamiento (rumiación, evitación experiencial), pero ninguno está adaptado para población oncológica y ninguno incluye los tres estilos de pensamiento (rumiación, evitación y compromiso cognitivo). Objetivo: diseñar y estudiar las propiedades psicométricas de un instrumento que mida estilos de pensamiento en respuesta al diagnóstico oncológico. Método: Estudio instrumental, dividido en dos fases: la primera, elaboración de reactivos y jueceo de expertos y la segunda, análisis de propiedades psicométricas. Resultados: La versión final cuenta 15 ítems. La estructura interna resultó de tres factores, obtenidos a partir de un Análisis Factorial Exploratorio y corroborada mediante un Análisis Factorial Confirmatorio. Los valores de alfa de cronbach de las escalas resultaron adecuadas: la de Rumiación resultó de 0,87 , la de Compromiso cognitivo de , 0,82 y de 0,72 la de evitación. Todos los ítems cuentan con evidencias de validez de contenido y propiedades psicométricas satisfactorias. La escala de Compromiso cognitivo fue la que obtuvo la puntuación media mayor y la de Rumiación mostró la menor. Conclusión: El Inventario de Estilos de Pensamiento en Respuesta al Cáncer (IEPRaC) cuenta con adecuadas propiedades psicométricas para ser utilizado en población oncológica mexicana.

Palabras clave: Estilos de pensamiento, respuestas cognitivas, evitación, rumiación, compromiso cognitivo, adaptación a la enfermedad.

1 Mariana Alejandra Sierra-Murguía. Cancer Center Tec100. Ignacio Zaragoza Pte 263 H16, col centro. Qro, Querétaro. México.

E-mail: msierra@cancercentertec100.com

2 Lucía González-Alcocer. Cancer Center Tec100. Ignacio Zaragoza Pte 263 H16, col centro. Qro, Querétaro. México.

E-mail: lukygoal22@gmail.com

3 Gabriela Navarro-Contreras. Universidad de Guanajuato Campus León. Depto. Psicología. Blvrd Cañaveral 1001, Fracciones de los Aguirre, 37672 León, Gto. México.

E-mail: gabriela.navarro.c@gmail.com

4 Martha Leticia Guevara-Sanginés. Universidad de Guanajuato. Fraccionamiento 1, Col. El Establo, Guanajuato, Gto. México

E-mail: leticiag@ugto.mx

5 Ferrán Padrós-Blázquez. Facultad de Psicología, Universidad Michoacana San Nicolás de Hidalgo; Morelia, Michoacán; México

E-mail: fpadros@uoc.edu

* Dirección de correspondencia: Mariana Alejandra Sierra-Murguía. Cancer Center Tec100. Ignacio Zaragoza Pte 263 H16, col centro. Qro, Querétaro. México. CP 76000. E-mail: msierra@cancercentertec100.com 
[en] Design and psychometric properties analysis of the Thought Style in Response to Cancer Inventory (IEPRaC)

\begin{abstract}
Thought style has been defined as one of the mediating variables to emotional response at a cancer diagnosis. It has been reported in pathological contexts (rumination in depression), but also in normative ones, it has been suggested that thought styles can predict adjustment to a stressor. There are measurement scales used to assess thought style independently (rumination, experiential avoidance), but none of them is adapted for oncologic population and none of them include all three thought styles. Aim: To design and assess psychometric properties of a scale of thought styles in response to a cancer diagnosis. Method: Instrumental Study, divided in two phases: the first, item elaboration and expert judge; the second, psychometric properties analysis. Results: Final version counts with 15 items. Intern Structure resulted in three factors, obtained by an Exploratory Factorial Analysis and corroborated by a Confirmatory Factorial Analisis. Cronbach's alphas for the subscales resulted appropriate: for rumination 0.87 , for cognitive engagement 0.82 and 0.72 for avoidance. All items have evidence for content validity and adequate psychometric properties. Results of Cognitive Engagement subscale had the higher mean and Rumination subscale was the lowest. Conclusion: Thought style in response to a cancer diagnosis scale (IERPaC) has adequate psychometric properties to be used in Mexican oncologic patients.
\end{abstract}

Keywords: Thought styles, cognitive response, cognitive avoidance, rumination, cognitive engagement, adaptation to cancer.

Sumario: 1 Introducción 2. Método 2.1. Fase 1. Elaboración de ítems y jueceo por expertos 2.2. Fase 2. Aplicación, validez de constructo, consistencia interna y depuración del cuestionario 3. Resultados 4. Discusión/ Conclusiones 5. Referencias bibliográficas.

Cómo citar: Sierra-Murguía MA, González-Alcocer L, Navarro-Contreras G, Guevara-Sanginés ML, Padrós-Blázquez F. Diseño y análisis de propiedades psicométricas del Inventario de Estilos de Pensamiento en Respuesta al Cáncer (IEPRaC). Psicooncología 2021; 18: 77-90. doi: 10.5209/ psic. 74533

\title{
1. Introducción
}

La experiencia del cáncer involucra un grupo de enfermedades crónicas, debilitantes y con riesgo letal. La persona debe enfrentarse, además de la propia enfermedad y los síntomas de la misma, a una serie de eventos aversivos como el diagnóstico, tratamiento y los efectos secundarios de los $\operatorname{mismos}^{(1)}$. Esto conlleva frecuentemente a provocar una experiencia de estrés, malestar emocional (distrés), depresión, ansiedad, incertidumbre, dificultades en el ajuste a la enfermedad y una disminución en las interacciones sociales ${ }^{(2)}$. En el paciente con cáncer, los eventos de vida estresantes y el trauma han sido asociados a un mayor riesgo de progresión de la enfermedad, además de mayor mortalidad ${ }^{(3)}$.

Por otro lado, también se ha descrito la presencia de cambios adaptativos positivos después de eventos potencialmente traumáticos como el cáncer ${ }^{(4)}$. Algunos de estas modificaciones se han agrupado en un constructo denominado crecimiento postraumático (CPT), referido a cambios positivos ocurridos después de una situación potencialmente traumática, por ejemplo, cambios en la percepción de uno mismo, apertura a nuevas posibilidades, cambios en las relaciones con otros, cambios en la apreciación de la vida y en espiritualidad ${ }^{(5)}$.

Una de las variables que ha sido definida como mediadora para la respuesta emocional ante el cáncer es el estilo de pensamiento, referido a la manera en 
que se presentan pensamientos sobre un estresor o conjunto de estresores, independientemente del contenido. Se ha reportado la relavancia de los estilos de pensamientos en contextos patológicos (por ejemplo, rumiación en depresión ${ }^{(6,12)}$, pero también en contextos normativos (por ejemplo, pensamientos asociados a la solución de un problema $)^{(7)}$ y se ha sugerido que pueden predecir el ajuste ante un estresor $^{(8)}$, en función del estilo de pensamiento: evitación cognitiva, rumiación o compromiso cognitivo.

La evitación cognitiva se refiere a las acciones que una persona realiza con el objetivo de impedir la exposición a pensamientos que generan malestar emocional. Este concepto es similar al de "Evitación experiencial"(9) definido como "fenómeno que ocurre cuando una persona no está dispuesta a ponerse en contacto con experiencias privadas particulares e intenta alterar la forma o frecuencia de esos eventos y el contexto que los ocasiona" (p. 1156). Es desadaptativa a mediano y largo plazo, ya que no permite que la persona enfrente la situación amenazante, no permite el procesamiento emocional, obstaculiza la solución de problemas o la búsqueda de sentido. Sin embargo, la adopción y mantenimiento del estilo evitativo reduce la ansiedad, lo cual supone un importante reforzamiento inmediato ${ }^{(10)}$. Pero a medio y largo plazo, la evitación cognitiva imposibilita que se dé un afrontamiento adaptativo frente a una experiencia amenazadora y se asocia a un incremento de síntomas físicos y psicológicos en pacientes con cáncer ${ }^{(11)}$.

La rumiación es otro estilo de pensamiento, el cual consiste en pensamientos intrusivos y repetitivos que generan malestar emocional de forma inmediata. La rumiación es considerada un estilo de pensamiento predictor y exacerbador de psicopatología ${ }^{(12)}$. Se ha señalado que los pensamientos repetitivos rumiativos interfieren con la capacidad de solución de problemas y posibles mejorías en el estado anímico ${ }^{(12)}$.

Otro estilo de pensamiento es el compromiso cognitivo, tipificado por pensamientos que generan cambios positivos en la evaluación cognitiva, búsqueda de sentido y soluciones a los problemas ${ }^{(8)}$.

En particular, en el modelo de crecimiento postraumático ${ }^{(13)}$, se alude al término compromiso cognitivo haciendo énfasis en que es un pensamiento repetitivo, que no necesariamente es intrusivo, ni negativo, y que incluye recuerdos, búsqueda de solución de problemas e intentos por encontrar un sentido a la situación estresante $^{(14)}$. Los pensamientos enfocados en búsqueda de sentido han sido descritos como una estrategia de afrontamiento efectiva para superar experiencias de vida estresantes ${ }^{(15)}$. Este tipo de pensamientos se han asociado a un crecimiento derivado de una experiencia potencialmente traumática, especialmente cuando se verbalizan o comparten con otras personas, en lugar de ser procesados en aislamiento $^{(8)}$.

Se ha descrito que la evaluación subjetiva que hace una persona sobre el diagnóstico de cáncer incide en mayor medida en el $\mathrm{CPT}$, que una variable más objetiva, como puede ser el estadío de la enfermedad ${ }^{(15,16)}$. Esto es congruente con el modelo de CPT, debido a que éste menciona que si una persona no evalúa el evento como potencialmente amenazante, entonces no será motivado a reevaluar su vida y a realizar cambios ${ }^{(13)}$. Para que se genere CPT se requiere que un evento sea suficientemente amenazador, que ponga en duda las creencias sobre el mundo, el sí mismo, sus metas y valores. A su vez que a partir de este evento, se genere pensamiento reflexivo y enfocado en búsqueda de sentido ${ }^{(13)}$. 
En cuanto a la evaluación de estilos de pensamientos se encuentra la Escala de Respuestas Rumiativas $^{(12)}$, que es una escala de 22 ítems y mide el grado en que un individuo se involucra en pensamientos rumiativos y es un indicador de la rumiación como rasgo, cuenta con tres subescalas de acuerdo al tipo de rumiación: reflexiva, melancólica y depresiva. Se sugiere que esta escala está relacionada con el estado clínico del paciente, en lugar de medir diferencias individuales no patológicas (en el estilo de pensamiento $)^{(17)}$. Una de las críticas que se ha hecho a esta escala es que no es útil para medir puramente rumiación como proceso cognitivo, ya que se ha hallado una elevada relación con el trastorno depresivo mayor ${ }^{(18)}$.

En cuanto a la evitación experiencial, existe el Cuestionario de Acción y Aceptación (AAQ) diseñado por Hayes et al.(19), del cual más adelante se hizo una segunda versión del instrumento (AAQ-II) ${ }^{(20)}$ que con mejores propiedades psicométricas.

Ninguna de las escalas mencionadas ha sido diseñada o adaptada para uso en pacientes oncológicos. Es por ello que el objetivo del presente estudio fue diseñar y estudiar las propiedades psicométricas de un instrumento que evalúe los estilos de pensamiento (evitación cognitiva, rumiación y compromiso cognitivo) relacionados con el cáncer, para pacientes oncológicos mexicanos. Por otro lado, la presente escala conceptualiza el estilo de pensamiento como una respuesta que surge frente a un diagnóstico oncológico, de tal manera que identificar el estilo de pensamiento de los pacientes en este contexto permitirá implementar intervenciones enfocadas específicamente en ellos para promover una mayor adaptación a la enfermedad y mejor calidad de vida.

\section{Método}

Este estudio está dividido en dos fases. En la primera, se realizó la elaboración de reactivos y el jueceo de expertos del Inventario de Estilos de Pensamiento en Respuesta al Cáncer (IEPRaC) (ver el anexo). La segunda fase consistió de la aplicación a una muestra de pacientes oncológicos y el análisis de propiedades psicométricas del IEPRaC.

\subsection{Fase 1. Elaboración de ítems y jueceo por expertos}

Objetivo fase 1. Jueceo y revisión de los ítems, para determinar validez de contenido y comprensión de los reactivos (pertinencia y redacción).

Procedimiento: En un estudio previo realizado por el mismo equipo de investigación se analizaron los contenidos y estilos de pensamiento que presentaba un grupo de pacientes oncológicos, a partir de los resultados de ese estudio se seleccionaron los contenidos y lenguaje para la redacción de ítems del IEPRaC.

Se redactaron 38 ítems a partir del siguiente plan de prueba: Se les explicó a los jueces sobre el constructo y las dimensiones que se busca medir, se les pidió que calificaran del 0-5 cada ítem acorde a pertinencia y redacción. A su vez se les solicitó sugerencias sobre los mismos.

Participantes fase 1: Para el jueceo de la primer versión del instrumento participaron ocho psicólogos que tenían algún posgrado en psicología de la salud o medicina conductual. 
Instrumentos: Formato diseñado para jueceo de expertos del instrumento y el IEPRaC, diseñado para el presente estudio.

Análisis: Se utilizó el coeficiente de Validez $\mathrm{V}$ de Aiken $^{(21)}$, que es la razón de un dato obtenido sobre la suma máxima de la diferencia de los valores posibles. Este coeficiente puede obtener valores de 0-1; entre más alto sea, mayor será la validez de contenido. La fórmula que utiliza es la siguiente:

$\mathrm{V}=\mathrm{S} /(\mathrm{n}(\mathrm{c}-1))$

$\mathrm{S}=$ sumatoria de las puntuaciones brindadas por cada juez

$\mathrm{n}=$ número de jueces $(8)$

$\mathrm{c}=$ número de posibilidades de los valores de la escala de calificación (6)

El criterio que utilizamos en el presente análisis fue de 0,8 ; es decir, los ítems que tuvieran un índice Aiken menor a 0,8 debían ser eliminados o modificados.

Resultados jueceo de expertos (fase 1): Después de obtener el índice Aiken para cada ítem (para pertinencia y para redacción), se obtuvieron los siguientes resultados: en la primer escala (Rumiación), se consideró que la redacción de tres ítems debía ser modificada. En la escala de compromiso cognitivo, no hubo ítems que tuvieran menos de 0,8 en índice Aiken, por lo que se consideraron pertinentes y con adecuada redacción. El mismo caso se presentó con la escala de evitación.

Se agregaron preguntas a sugerencia de los jueces, quedando una nueva versión con 38 ítems para ser estudiada y depurada en función de criterios psicométricos.

\subsection{Fase 2. Aplicación, validez de constructo, consistencia interna y depuración del cuestionario}

Objetivo: Estudiar la bondad de los reactivos, la consistencia interna de las escalas y la validez de constructo del IEPRaC.

Participantes: Tomando en cuenta que son 38 ítems, calculando la muestra con cinco sujetos por ítem ${ }^{(22)}$, da un total de 190 participantes para esta fase del estudio. Pero se consigieron hasta 235 participantes de la Ciudad de Querétaro, México, de los cuales 135 (57,44\%) fueron mujeres. La media de edad fue de 50,6, con un rango de 18-83 años y DT de 13,59. Los diagnósticos oncológicos que tenían los participantes fueron cáncer de mama $94(40,00 \%)$, de sistema nervioso central 5 $(2,12 \%)$, renal $3(1,27 \%)$, de próstata $13(5,53 \%)$, hematológico $18(7,65 \%)$, gástrico $21(8,93 \%)$, urológico $19(8,08 \%)$, ginecológico $32(9,84 \%)$, cabeza y cuello 9 $(3,82 \%)$, de pulmón $6(2,55 \%)$ y otro $15(6,38 \%)$.

Instrumento: Versión resultante del jueceo por expertos del IEPRaC

Procedimiento: Debido a la contingencia sanitaria resultante de la pandemia de COVID 19 el acceso al centro oncológico fue restringido, por lo que se adaptó el cuestionario a una versión electrónica utilizando Google Forms donde también se solictó información sobre la edad, sexo y tipo de cáncer. Éste se compartió a los pacientes de un centro oncológico privado de la Ciudad de Querétaro (México) con ayuda del departamento de relaciones públicas y de grupos de apoyo oncológicos.

Confidencialidad de los datos y consentimiento informado: Al principio del formato electrónico se explicaba sobre los objetivos y procedimientos del estudio y que se garantizaría la confidencialidad de sus datos. El consentimiento se firmó por escrito en el mismo formato de Google Forms. 
Análisis de datos. Se analizaron los datos sociodemográficos utilizando estadística descriptiva (media, desviación típica, asimetría y curtosis para variables cuantitativas y frecuencias y porcentajes para variables cualitativas). Se realizó un análisis de discriminación de ítems por medio de la t de Student, del cual se eliminaron tres ítems ya que no discriminaron.

Se hizo un análisis factorial exploratorio por el método de máxima verosimilitud con rotación equarmax debido a que a que se recomienda cuando se piensa en factores no correlacionados. La rotación equarmax combina el método varimax, que simplifica los factores, y el método quartimax, que simplifica las variables ${ }^{(22)}$. Se minimiza tanto el número de variables que saturan alto en un factor, como el número de factores necesarios para explicar una variable. Además de la prueba de esfericidad de Bartlett y Medida de adecuación muestral de Kaiser-Meyer-Olkin (KMO). Posteriormente, se hizo análisis de Alfa de Cronbach por cada escala, no se hizo para la escala total debido a que se consideran factores independientes.

También, se aplicó un Análisis Factorial Confirmatorio (AFC) a través de los Mínimos Cuadrados Ponderados Robustos debido a que se considera una aproximación apropiada para el AFC con datos categóricos ${ }^{(23)}$.Tomando en consideración a Hooper, Coughlan y Mullen ${ }^{(24)}$ se consideraron los siguientes índices de ajuste: la chi-cuadrada de Satorra-Bentler $\left(\chi^{2}\right)$ y grados de libertad, de los cuales se infiere un ajuste adecuado, cuando el valor de $\mathrm{p}$ extraído de $\chi^{2}$ resulta no significativo, asimismo cuando $\chi^{2} /$ $g l<3$, se interpreta como excelente y $<5$ aceptable ${ }^{(25)}$. También se consideraron los siguientes; índice de ajuste normado (NFI), Tucker-Lewis (TLI) y comparativo (CFI), los valores se interpretan como excelente ajuste cuando son superiores a 0,95 y adecuados por encima de $0,90^{(26)}$. Asimismo, se estimó el error cuadrático medio de aproximación (RMSEA), valores inferiores a 0,08 , indican buen ajuste ${ }^{(27)}$.

\section{Resultados}

En el Análisis Factorial exploratorio se obtuvo una adecuación muestral de KMO $=0,854$, y la prueba de esfericidad de Bartlett de $\chi^{2}=4082,438, \mathrm{gl}=703$, resultó significativa $(\mathrm{p}<0,001)$. Esto indica que es adecuado proceder con el análisis factorial. Del análisis de reducción de dimensiones, resultaron 3 dimensiones que explican el $45,05 \%$ de la varianza total. Se tomaron como criterios; autovalores superiores a la unidad, diferencia notable entre autovalores y que los factores resultaran congruentes y etiquetables. Se eliminaron un total de 3 ítems, porque mostraron pesos factoriales menores a 0,30 en las dimensiones obtenidas o saturaban en más de un factor. Con la versión de 35 ítems también se obtuvieron indicadores que hacen adecuado el análisis factorial $\left(\mathrm{KMO}=0,862 ; \chi^{2}=3746,134, \mathrm{gl}=595 ; \mathrm{p}<0,001\right)$, la mejor solución también resultó de tres factores; Rumiación (17 ítems), Compromiso cognitivo (13 ítems) y Evitación (5 ítems) y explicó un 46,923\% de la varianza. Tomando en cuenta el reducido número de ítems del factor Evitación, se propuso estudiar una versión reducida de 5 ítems por escala (escogiendo los reactivos que mostraron mayores saturaciones de las escalas de Rumiación y de Compromiso Cognitivo).

En el análisis factorial exploratorio de la versión de 15 ítems, la prueba de esfericidad de Bartlett resultó también significativa $\left(\chi^{2}=1221,460, \mathrm{gl}=105 ; \mathrm{p}<\right.$ $0,001)$ y el test $\mathrm{KMO}=0,808$, lo cual indicó la aplicabilidad del análisis factorial a los datos. Se matuvo la solución de 3 factores que explicaban el 58,9\% de la varianza. 
En la tabla 1 se muestra que el tercer componente obtiene un autovalor superior a 2 , y se observa una gran diferencia respecto al autovalor del cuarto componente que además resultó inferior a la unidad.

Tabla 1. Valores propios, varianza explicada y acumulada de los factores del IEPRaC.

\begin{tabular}{cccc}
\hline Componente & Autovalor & $\begin{array}{c}\text { \% Varianza } \\
\text { explicada }\end{array}$ & $\begin{array}{c}\text { \% Varianza } \\
\text { acumulado explicada }\end{array}$ \\
\hline $\mathbf{0 1}$ & $\mathbf{3 , 7 5 0}$ & $\mathbf{2 5 , 0 0 0}$ & $\mathbf{2 5 , 0 0 0}$ \\
$\mathbf{0 2}$ & $\mathbf{2 , 7 8 1}$ & $\mathbf{1 8 , 5 4 2}$ & $\mathbf{4 3 , 5 4 2}$ \\
$\mathbf{0 3}$ & $\mathbf{2 , 3 1 0}$ & $\mathbf{1 5 , 3 9 7}$ & $\mathbf{5 8 , 9 3 9}$ \\
04 &, 909 & 6,058 & 64,997 \\
05 &, 762 & 5,083 & 70,080 \\
\hline
\end{tabular}

Por otro lado, en la tabla 2, puede observase que las saturaciones de los ítems de la versión final resultaron todas superiores a 0,40 , y sólo saturaron en un factor.

Tabla 2. Cargas factoriales de los 15 reactivos del IEPRaC en los tres factores; Rumiación (1), Compromiso cognitivo (2) y Evitación (3).

\begin{tabular}{|c|c|c|c|}
\hline & \multicolumn{3}{|c|}{ Componente } \\
\hline & 1 & 2 & 3 \\
\hline 9. Pienso en lo triste que me siento por el cáncer & 841 & & \\
\hline 6. Pienso en lo mal que me siento por el cáncer & ,838 & & \\
\hline 14. Pienso en el daño que me produce el cáncer & 824 & & \\
\hline 13. Pienso cosas malas de mi enfermedad & ,808 & & \\
\hline 1. Pienso en lo injusto que es el cáncer & ,751 & & \\
\hline 12. Pienso en lo que esta enfermedad me ha enseñado & & 802 & \\
\hline $\begin{array}{l}\text { 2. Pienso en que esta enfermedad me ha unido más a mis seres } \\
\text { queridos }\end{array}$ & & ,774 & \\
\hline 7. Pienso que he crecido en otras áreas desde que me enfermé & & ,773 & \\
\hline 4. Pienso en lo que he aprendido de tener cáncer & &, 738 & \\
\hline 15. Pienso que si supero esto, seré más fuerte & & 698 & \\
\hline $\begin{array}{l}\text { 5. Intento evitar sentimientos desagradables derivados del } \\
\text { cáncer }\end{array}$ & & & ,747 \\
\hline 3. Trato de pensar en otras cosas, para no pensar en el cáncer & & & ,738 \\
\hline 10. Intento evitar pensar en el cáncer & & & ,715 \\
\hline 8. Trato de evitar pensar sobre la muerte & & & ,645 \\
\hline 14. Trato de no preocuparme por el cáncer & & &, 565 \\
\hline
\end{tabular}

Nota: se presentan los valores superiores a 0,30 .

Respecto al AFC, el valor de chi-cuadrado de Satorra-Bentler $\left(\chi^{2}\right)$ fue de 174,742 con 89 grados de libertad resultó significativo $\mathrm{p}<0,001$ y el índice NFI resultó 0,861 , ambos indican mal ajuste. Sin embargo, el valor de $\chi^{2} / g l$ resultó $<3$, lo cual se interpreta como excelente ajuste. Además los índices TLI y CFI y RMSEA también resultaron adecuados. 
Tabla 3. Índices de bondad de ajuste para el modelo de tres factores del IEPRaC de 15 ítems.

\begin{tabular}{llllllll}
\hline & $\chi^{2}(\mathrm{gl})$ & $\mathrm{p}$ & $\chi^{2} / \mathrm{gl}$ & NFI & TLI & CFI & RMSEA \\
\hline $\begin{array}{l}\text { Versión } \\
15 \text { ítems }\end{array}$ & $174,742(89)$ & $<, 001$ & 1,963 &, 861 &, 912 &, 925 &, 066 \\
IEPRaC & & & & & & & \\
\hline
\end{tabular}

Nota: Índices corregidos (método robusto). S-B $\chi 2=\chi 2$ escalado de Satorra-Bentler, dividido entre grados de libertad; NFI = índice de ajuste normalizado; TLI = Índice de Tucker-Lewis CFI = índice de bondad de ajuste comparativo; RMSEA = Índice de error de aproximación cuadrático medio.

En la figura 1 se observa el modelo del análisis factorial confirmatorio, con los tres factores teóricos.

Figura 1. Estimación de los parámetros del modelo propuesto de 3 factores del IEPRaC.
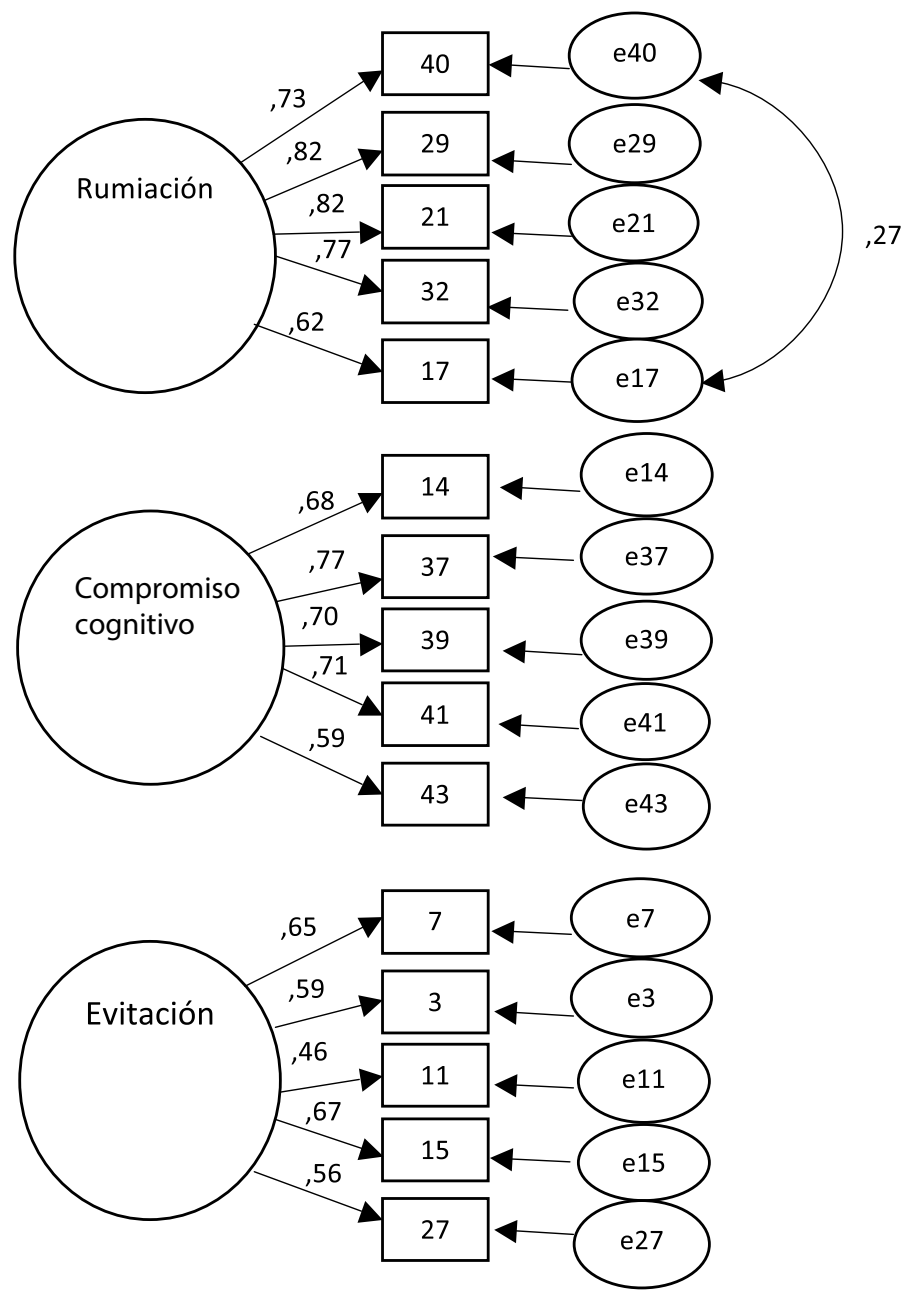
Respecto a la consistencia interna del IEPRaC, se obtuvieron alfas de Cronbach aceptables en las tres escalas; rumiación $(\alpha=0,871)$, compromiso cognitivo ( $\alpha$ $=0,819)$ y evitación $(\alpha=0,717)$. Como puede observarse en la tabla 3 , todos los reactivos al ser eliminados hacen reducir el valor del Alfa de Cronbach de la escala a la que pertenecen. Además todos los ítems muestran correlaciones corregidas con la escala total superiores a 0,40 , a excepción del ítem 14 que pertenece a la escala de evitación que resultó de 0,368 .

Los valores de las medias ariméticas de los ítems de la escala de Evitación están alrededor de 2, por encima de 2 los ítems de la de Compromiso Cognitivo, sin embargo las medias de los ítems de la escala de Rumiación se mostraron alrededor de 1. Los valores de las desviaciones típicas de todos los reactivos resultaron cercanos a la unidad. Finalmente los valores de asimetría y curtosis sugieren que los la distribución de los ítems es cercana a la normalidad (ver tabla 4).

Tabla 4. Resultados análisis de consistencia interna de las escalas Rumiación, Evitación y Compromiso cognitivo.

\begin{tabular}{|c|c|c|c|c|c|c|}
\hline & M & DT & Sk & $\mathrm{K}$ & $\begin{array}{l}\text { r ítem con } \\
\text { total de } \\
\text { elementos } \\
\text { corregida }\end{array}$ & $\begin{array}{l}\text { Cambio de } \\
\text { Alfa de si } \\
\text { elemento se } \\
\text { suprime }\end{array}$ \\
\hline \multicolumn{7}{|c|}{ Escala de Rumiación } \\
\hline 1 & 1,15 & 1,13 & 0,457 & $-1,220$ & 623 &,- 004 \\
\hline 6 & 1,04 & 0,93 & 0,601 & $-0,482$ &, 728 &,- 035 \\
\hline 9 & 0,96 & 0,93 & 0,635 & $-0,523$ & ,728 &,- 035 \\
\hline 11 & 1,22 & 0,98 & 0,289 & $-0,947$ & ,707 &,- 030 \\
\hline 13 & 0,95 & 0,89 & 0,768 & $-0,031$ &, 724 &,- 033 \\
\hline \multicolumn{7}{|c|}{ Escala de de Evitación } \\
\hline 3 & 2,01 & 0,91 & $-0,661$ & $-0,341$ & ,541 &,- 074 \\
\hline 5 & 2,18 & 0,89 & $-1,001$ & 0,329 & ,565 &,- 082 \\
\hline 8 & 1,90 & 0,97 & $-0,505$ & $-0,728$ & ,442 &,- 034 \\
\hline 10 & 1,80 & 0,92 & $-0,352$ & $-0,680$ & ,473 &,- 047 \\
\hline 14 & 1,96 & 0,96 & $-0,638$ & $-0,525$ & 368 &,- 004 \\
\hline \multicolumn{7}{|c|}{ Escala de Compromiso cognitivo } \\
\hline 2 & 2,28 & 0,90 & $-1,049$ & 0,129 & 625 &,- 040 \\
\hline 4 & 2,21 & 0,87 & $-1,078$ & 0,637 &, 599 &,- 032 \\
\hline 7 & 2,05 & 0,86 & $-0,653$ & $-0,178$ & 630 &,- 041 \\
\hline 12 & 2,19 & 0,87 & $-0,995$ & 0,440 & 667 &,- 052 \\
\hline 15 & 2,48 & 0,85 & $-1,649$ & 1,873 &, 533 &,- 014 \\
\hline
\end{tabular}

En la tabla 5 se muestran los valores de media aritmética, desviación típica, asimetría y curtosis de las puntuaciones totales de las escalas del IEPRaC. La tabla 5 permite comparar las puntuaciones, y se observa un valor promedio de la escala de rumiación menor respecto a las escalas de Evitación y Compromiso Cognitivo, esta última fue la que mostró la mayor media aritmética. Los valores de la desviación típica de las tres escalas fueron superiores a 3 y menores a 4 . Los valores de asimetría 
y curtosis de las puntuaciones totales sugieren que las tres escalas se distribuyen siguiendo la ley normal.

También en la tabla 5 se puede observar como la mayoría de los participantes obtiene puntuaciones bajas en la escala de rumiación, en la mitad alta en la de evitación y puntuaciones elevadas en la escala de Compromiso Cognitivo. Debe señalarse que los participantes se ubican en todo el rango posible de puntuaciones finales de las tres escalas.

Tabla 5. Media, desviación típica, asimetría, curtosis, frecuencias y porcentajes de los valores de la puntuación total de las escalas del IEPRaC.

\begin{tabular}{|c|c|c|c|c|c|c|}
\hline & \multicolumn{2}{|c|}{ Rumiación } & \multicolumn{2}{|c|}{ Evitación } & \multicolumn{2}{|c|}{ Compromiso Cognitivo } \\
\hline & $M=5,31$ & $\mathrm{Sk}=0,53$ & $M=9,84$ & $\mathrm{Sk}=-0,57$ & $\mathrm{M}=11,20$ & $\mathrm{Sk}=-1,09$ \\
\hline & $\mathrm{DT}=3,95$ & $K=-0,43$ & $\mathrm{DT}=3,19$ & $\mathrm{~K}=0,23$ & $\mathrm{DT}=3,31$ & $\mathrm{~K}=1,28$ \\
\hline Valores & Frecuencia & $\%$ & Frecuencia & $\%$ & Frecuencia & $\%$ \\
\hline 0 & 31 & 13,8 & 2 & 0,9 & 4 & 1,8 \\
\hline 1 & 14 & 6,2 & 1 & 0,4 & 0 & 0,0 \\
\hline 2 & 20 & 8,9 & 3 & 1,3 & 1 & 0,4 \\
\hline 3 & 16 & 7,1 & 4 & 1,8 & 3 & 1,3 \\
\hline 4 & 17 & 7,6 & 4 & 1,8 & 2 & 0,9 \\
\hline 5 & 27 & 12,0 & 7 & 3,1 & 3 & 1,3 \\
\hline 6 & 23 & 10,2 & 12 & 5,3 & 6 & 2,7 \\
\hline 7 & 22 & 9,8 & 12 & 5,3 & 9 & 4,0 \\
\hline 8 & 9 & 4,0 & 24 & 10,7 & 14 & 6,2 \\
\hline 9 & 11 & 4,9 & 24 & 10,7 & 14 & 6,2 \\
\hline 10 & 9 & 4,0 & 33 & 14,7 & 25 & 11,1 \\
\hline 11 & 3 & 1,3 & 30 & 13,3 & 22 & 9,8 \\
\hline 12 & 6 & 2,7 & 23 & 10,2 & 34 & 15,1 \\
\hline 13 & 10 & 4,4 & 14 & 6,2 & 22 & 9,8 \\
\hline 14 & 4 & 1,8 & 19 & 8,4 & 26 & 11,6 \\
\hline 15 & 3 & 1,3 & 13 & 5,8 & 40 & 17,8 \\
\hline
\end{tabular}

Respecto a la relación entre las escalas, no se observaron correlaciones significativas entre ninguna de las escalas (Rumiación con evitación fue de $\mathrm{r}=$ $-0,118 ; \mathrm{p}=0,079$, Rumiación con Compromiso Cognitivo de $\mathrm{r}=-0,080 ; \mathrm{p}=0,232$ y evitación con compromiso cognitivo de $r=0,110 ; p=0,100$ ).

\section{Discusión/ Conclusiones}

El objetivo de este estudio fue diseñar y estudiar las propiedades psicométricas de un instrumento que mida estilos de pensamientos en respuesta al cáncer. Después del jueceo se concluye que el Inventario de Estilos de Pensamiento en Respuesta 
al Cáncer IEPRaC cuenta con evidencias de validez de contenido. Respecto a la validez de constructo, el análisis factorial exploratorio sugiere una estructura de tres factores coincidiendo con la propuesta teórica; Rumiación, Evitación y Compromiso Cognitivo. Sin embargo, en el análisis factorial confirmatorio el ajuste resultó dudoso.

Por otro lado, los índices de consistencia interna mostrada por las tres escalas fueron adecuados. Los 15 ítems que componen la versión final, muestran excelentes características psicométricas, con excepción del ítem 14 que pertenece a la escala de evitación, cuyo valor de correlación corregida es solo aceptable, pero el resto de indicadores son adecuados.

Las puntuaciones totales de las tres escalas se distribuyen siguiendo la ley normal, y en las tres escalas se observa que algunos participantes obtuvieron puntuaciones extremas. Es importante mencionar la ausencia de relaciones significativas entre las tres escalas, lo cual sugiere que son estilos de respuesta cognitiva claramente diferenciados. Se observan puntuaciones menores en la escala de rumiación, y la de mayor puntuación media fue la de compromiso cognitivo. Lo cual indica la presencia en mayor frecuencia de estilos de pensamiento adaptativo ${ }^{(8,28)}$. Sin embargo, puede haber influido la deseabilidad social, variable que no ha sido controlada en la presente investigación y sería conveniente controlarla en futuros estudios.

Una limitación importante de la presente investigación se refiere al tipo de muestreo utilizado, el cual fue realizado por conveniencia y sólo fue obtenido de un centro médico privado, de modo que las características de la muestra estudiada no son representativas de la población mexicana. Sería conveniente replicar el estudio haciéndo uso de muestras más amplias, de diferentes estratos socio-económicos y de varios estados de la república.

Otra limitación es la ausencia de evidencias de validez concurrente, en un futuro sería pertinente estudiar la relación de las escalas con instrumentos genéricos como la escala de rumiación ${ }^{(6,12)}$ y la de evitación ${ }^{(19,20)}$. Asimismo, sería de utilidad estudiar la relación de las tres escalas con mediciones de sintomatología depresiva y de ansiedad, así como de calidad de vida o bienestar.

Por otro lado, en el presente estudio tampoco se estudió la estabilidad temporal de las escalas, nótese que al estar evaluando estilos de respuesta cognitiva, éstos se espera que en ausencia de intervención no se modifiquen demasiado. En futuros estudios sería necesario estudiar la fiabilidad test-retest de las escalas, y también sería conveninente estimar la sensibilidad al cambio, en participantes antes y después de recibir una intervención exitosa.

La versión final del IEPRaC es intencionalmente breve pues es recomendable para pacientes oncológicos debido a la fatigabilidad que pueden presentar y que requieren tratamiento ${ }^{(29)}$.

El IEPRaC puede ser de gran utilidad en el ámbito aplicado para detectar aquellos individuos que presentan una respuesta cognitiva que implique un factor de riesgo, como altos niveles de rumiación o evitación y beneficiarse de intervenciones psicológicas. Además de la utilidad en el ámbito de la investigación, recuérdese que el estilo de respuesta cognitiva (rumiación, evitación cognitiva, compromiso cognitivo) es una variable que ha sido descrita como mediadora para la respuesta emocional a un estresor identificado ${ }^{(17)}$.

Puede concluirse que el IEPRaC es un instrumento breve que muestra adecuadas propiedades psicométricas para ser utilizado en población oncológica mexicana. 


\section{Referencias bibliográficas}

1. NCCN. Distress management. Natl Compr Cancer Netw Guidel. 2020; Version 2.

2. Grassi L, Spiegel D, Riba M. Advancing psychosocial care in cancer patients. F1000Research. 2017;6(0). doi: 10.12688/f1000research.11902.1

3. Antoni MH, Dhabhar FS. The impact of psychosocial stress and stress management on immune responses in patients with cancer. Cancer 2019;125:1417-31. doi: 10.1002/ cncr.31943

4. Bronwyn Morris Senior, Shakespeare- Finch J. Rumination, post-traumatic growth, and distress : Structural equation modelling with cancer survivors. Psychooncology 2011; 20:1176-83 doi: 10.1002/pon.1827

5. Calhoun LG. Facilitating posttraumatic growth: A Clinician's guide (Personality and Clinical Psychology Series) [Internet]. Routledge; 1999 [Acceso 18 de noviembre de 2018]. Disponible en: http://www.amazon.com/Facilitating-Posttraumatic-GrowthClinicians-Personality/dp/080582412X

6. McLaughlin KA, Nolen-Hoeksema S. Rumination as a transdiagnostic factor in depression and anxiety. Behav Res Ther 2011;49:186-93. doi: 10.1016/j.brat.2010.12.006

7. DuPre. Spontaneous thought gets stuck with rumination.pdf [Internet]. 2017 Acceso 18 de noviembre de 2019]. Disponible en: https://www.researchgate.net/publication/312448333

8. Segerstrom SC, Stanton AL, Alden LE, Shortridge BE. A multidimensional structure for repetitive thought: what's on your mind, and how, and how much? J Pers Soc Psychol 2003; 85: 909-21. doi: 10.1037/0022-3514.85.5.909.

9. Hayes SC, Wilson KG, Gifford E V., Follette VM, Strosahl K. Experiential avoidance and behavioral disorders: A functional dimensional approach to diagnosis and treatment. J Consult Clin Psychol 1996;64:1152-68.

10. Rauch S, Foa E. Emotional processing theory (EPT) and exposure therapy for PTSD. J Contemp Psychother 2006;36:61-5.

11. Bauer MR, Wiley JF, Weihs KL, Stanton AL. Stuck in the spin cycle: Avoidance and intrusions following breast cancer diagnosis. Br J Health Psychol 2017;22:609-26. doi: $10.1111 /$ bjhp. 12252

12. Treynor W, Gonzalez R, Nolen-Hoeksema S. Rumination Reconsidered: a psychometric analysis. Cognit Ther Res 2003;27:247-59.

13. Calhoun LG, Tedeschi RG. Handbook of posttraumatic growth: Research and Practice. Lawrence Erlbaum Associates, Publihers. 2006.

14. Martin LL, Tesser A. Ruminative Thoughts. In: Ruminative Thoughts: Advances in Social Cognition, Volumen IX. 1996.

15. Kamijo N, Yukawa S. ScienceDirect Meaning making and rumination: The roles of threat evaluation and personality. Procedia - Soc Behav Sci [Internet]. 2014;113:118-23. doi: 10.1016/j.sbspro.2014.01.017

16. Widows MR, Jacobsen PB, Booth-Jones M, Fields KK. Predictors of posttraumatic growth following Bbone marrow transplantation for cancer. Health Psychol 2005;24:266-73. doi:10.1037/0278-6133.24.3.266

17. DuPre E, Spreng RN. Spontaneous thought gets stuck with rumination.pdf. In: The Christoff K, Fox KCR, editors. Oxford handbook of spontaneous thought: Mindwandering, creativity, and dreaming. Oxford: Oxford University Press, 2018. 10.1093/ oxfordhb/9780190464745.013.5 
18. Smith TG. Reconciling psychology with economics: Obesity, behavioral biology, and rational overeating. J Bioeconomics [Internet]. 2009; 11:249-82. [Acceso 19 de enero de 2021]. Disponible en: http://link.springer.com/10.1007/s10818-009-9067-8

19. Hayes SC, Strosahl K, Wilson KG, Bisset R, Pistorello J, Toarmino D, et al. Measuring experiential avoidance: a preliminary test of a working model. Psychol Rec [Internet]. 2004;54:553-78. Disponible en: http://eprints.uanl.mx/5481/1/1020149995.PDF

20. Bond FW, Hayes SC, Baer RA, Carpenter KM, Guenole N, Orcutt HK, et al. Preliminary psychometric properties of the Acceptance and Action Questionnaire-II: A revised measure of psychological inflexibility and experiential avoidance. Behav Ther 2011;42:676-88. doi: 10.1016/j.beth.2011.03.007

21. Aiken LR. Content validity and reliability of single items or questionnaires. Educ Psychol Meas 1980;40:955-9.

22. López-Aguado M, Gutiérrez-Provecho L. Cómo realizar e interpretar un análisis factorial exploratorio utilizando SPSS. REIRE Rev d Innovació i Recer en Educ 2019;12 (2):1-14.

23. Muthén L, Muthén B. Mplus Version 7 user's guide. Los Angeles, CA Muthén Muthén. 2012;

24. Hooper D, Coughlan J, Mullen MR. Structural equation modelling: Guidelines for determining model fit. Electron J Bus Res Methods 2008;6:53-60.

25. Bollen KA. Structural equations with latent variables. New York: John Wiley \& Sons, Inc, 1989. p.1-9.

26. Hu L-T, Bentler PM. Cutoff criteria for fit indexes in covariance structure analysis: conventional criteria versus new alternatives. Struct Equ Model 1999; 6: 1-55. doi: 10.1080/10705519909540118

27. Kline R. Principles and practice of structural equation modeling. Third. Vol. 20, Thought. The Guilford Press; 2011.

28. Kamijo N, Yukawa S. Meaning making and rumination: The roles of threat evaluation and personality. Procedia - Soc Behav Sci [Internet]. 2014;113:118-23. doi: 10.1016/j. sbspro.2014.01.017

29. Mustian KM, Alfano CM, Heckler C, Kleckner AS, Kleckner IR, Leach CR, et al. Comparison of pharmaceutical, psychological, and exercise treatments for cancer-related fatigue: A meta-analysis. JAMA Oncol 2017;3:961-8. 


\section{ANEXO: Inventario de Estilos de Pensamiento en Respuesta al Cáncer (IEPRaC-15)}

INSTRUCCIONES: Por favor lee cuidadosamente las afirmaciones que se te presentan a continuación, responde lo primero que se te venga a la mente marcando con una $\mathbf{X}$ la opción con la que te identifiques; ya sea Nunca, Casi nunca, A menudo o Siempre.

\begin{tabular}{|l|l|l|l|l|}
\hline & Nunca & $\begin{array}{c}\text { Casi } \\
\text { Nunca }\end{array}$ & $\begin{array}{c}\text { A } \\
\text { menudo }\end{array}$ & Siempre \\
\hline $\begin{array}{l}\text { 1. Pienso en lo injusto que es el cáncer } \\
\text { 2. Pienso en que esta enfermedad me ha unido } \\
\text { más a mis seres queridos }\end{array}$ & & & & \\
\hline $\begin{array}{l}\text { 3.Trato de pensar en otras cosas, para no pensar } \\
\text { en el cáncer }\end{array}$ & & & & \\
\hline $\begin{array}{l}\text { 4. Pienso en lo que he aprendido de tener cáncer } \\
\text { 5. Intento evitar sentimientos desagradables } \\
\text { derivados del cáncer }\end{array}$ & & & & \\
\hline 6.Pienso en lo mal que me siento por el cáncer & & & & \\
\hline $\begin{array}{l}\text { 7. Pienso que he crecido en otras áreas desde que } \\
\text { enfermé }\end{array}$ & & & & \\
\hline 8. Trato de evitar pensar sobre la muerte & & & & \\
\hline 9. Pienso en lo triste que me siento por el cáncer & & & & \\
\hline 10. Intento evitar pensar en el cáncer & & & & \\
\hline 11. Pienso en el daño que me produce el cáncer & & & & \\
\hline $\begin{array}{l}\text { 12. Pienso en lo que esta enfermedad me ha } \\
\text { enseñado }\end{array}$ & & & & \\
\hline 13. Pienso cosas malas de mi enfermedad & & & & \\
\hline 14. Trato de no preocuparme por el cáncer & & & & \\
\hline 15. Pienso que si supero esto, seré más fuerte & & & & \\
\hline
\end{tabular}

Nota: Los ítems 1, 6 ,9,11 y 13 son de la escala Rumiación; los reactivos 3, 5 ,8, 10 y 14 son de la escala Evitación y los ítems 2, 4, 7, 12 y 15 de la escala Compromiso Cognitivo. 